\title{
EVA Swab Tool to Support Planetary Protection and Astrobiology Evaluations
}

\author{
Michelle A. Rucker \\ NASA Johnson Space Center \\ 2101 Nasa Parkway \\ Houston, TX 77058 \\ 281-244-5569 \\ michelle.a.rucker@nasa.gov
}

\author{
Drew Hood \\ NASA Johnson Space Center \\ 2101 Nasa Parkway \\ Houston, TX 77058 \\ 281-942-8858 \\ drew.hood@nasa.gov
}

\author{
Mary Walker \\ NASA Johnson Space Center \\ 2101 Nasa Parkway \\ Houston, TX 77058 \\ 281-244-2772 \\ mary.walker@nasa.gov
}

\author{
Dr. Kasthuri J. Venkateswaran \\ NASA Jet Propulsion Laboratory \\ 4800 Oak Grove Drive \\ Pasadena, CA 91109 \\ 818-393-1481 \\ kasthuri.j.venkateswaran@jpl.nasa.gov
}

\author{
Dr. Andrew C. Schuerger \\ University of Florida \\ 505 Odyssey Way, Exploration Park \\ Merritt Island, FL 32953 \\ 321-261-3774 \\ schuerg@ufl.edu
}

Keywords: Mars, Planetary Protection, Spacesuit, EVA, Microorganism, Astrobiology

\begin{abstract}
When we send humans to search for life on other planets, we'll need to know what we brought with us versus what may already be there. To ensure our crewed systems meet planetary protection requirementsand to protect our science from human contamination-we'll need to assess whether microorganisms may be leaking or venting from our spacecraft. Microbial sample collection outside of a pressurized spacecraft is complicated by temperature extremes, low pressures that preclude the use of laboratory standard (wetted) swabs, and operation either in bulky spacesuits or with robotic assistance. Engineers at the National Aeronautics and Space Administration (NASA) recently developed a swab kit for use in collecting microbial samples from the external surfaces of crewed spacecraft, including spacesuits. The Extravehicular Activity (EVA) Swab Kit consists of a single swab tool handle and an eight-canister sample caddy. The design team minimized development cost by re-purposing a heritage Space Shuttle tile repair handle that was designed to quickly snap into different tool attachments by engaging a mating device in each attachment. This allowed the tool handle to snap onto a fresh swab attachment much like popular shaving razor handles can snap onto a disposable blade cartridge. To disengage the handle from a swab, the user performs two independent functions, which can be done with a single hand. This dual operation mitigates the risk that a swab will be inadvertently released and lost in microgravity. Each swab attachment is fitted with commercially available foam swab tips, vendor-certified to be sterile for Deoxyribonucleic Acid (DNA). A microbial filter installed in the bottom of each sample container allows the container to outgas and repressurize without introducing microbial contaminants to internal void spaces. Extensive ground testing, post-test handling, and sample analysis confirmed the design is able to maintain sterile conditions as the canister moves between various pressure environments. To further minimize cost, the design team acquired extensive ground test experience in a relevant flight environment by piggy-backing onto suited crew training runs. These training runs allowed the project to validate tool interfaces with pressurized EVA gloves and collect user feedback on the tool design and function, as well as characterize baseline microbial data for different types of spacesuits. In general, test subjects found the EVA Swab Kit relatively straightforward to operate, but identified a number of design improvements that will be incorporated into the final design. Although originally intended to help characterize human forward contaminants, this tool has other potential applications, such as for collecting and preserving space-exposed materials to support astrobiology experiments.
\end{abstract}

